\title{
Effect of UV Ageing on the fatigue life of bulk polyethylene
}

\author{
Hamza Lamnii ${ }^{1,2}$, Moussa Nait-Abdelaziz ${ }^{1,{ }^{*}}$, Georges Ayoub ${ }^{2,3}$, Jean-Michel Gloaguen ${ }^{4}$, Ulrich Maschke ${ }^{4}$ and Bilal \\ Mansoor $^{3}$ \\ ${ }^{1}$ Universite de Lille, FRE 3723 - LML - Laboratoire de Mécanique de Lille, 59000 Lille, France \\ ${ }^{2}$ Mechanical Engineering Program, Texas A\&M University at Qatar, Education City, Qatar \\ ${ }^{3}$ Industrial and Manufacturing System Engineering department, University of Michigan Dearborn, 48128 Dearborn, USA \\ ${ }^{4}$ Université de Lille, Unité Matériaux Et Transformations (UMET), UMR CNRS 8207, F-59650 Villeneuve d'Ascq, France
}

\begin{abstract}
Polymers operating in various weathering conditions must be assessed for lifetime performance. Particularly, ultraviolet (UV) radiations alters the chemical structure and therefore affect the mechanical and fatigue properties. The UV irradiation alters the polymer chemical structure, which results into a degradation of the mechanical and fatigue behavior of the polymer. The polymer properties degradation due to UV irradiation is the result of a competitive process of chain scission versus post-crosslinking. Although few studied investigated the effect of UV irradiation on the mechanical behaviour of thermoplastics, fewer examined the UV irradiation effect on the fatigue life of polymers. This study focuses on investigating the effect of UV irradiation on the fatigue properties of bulk semi-crystalline polymer; the low density Polyethylene (LDPE). Tensile specimens were exposed to different dose values of UV irradiation then subjected to fatigue loading. The fatigue tests were achieved under constant stress amplitude at a frequency of $1 \mathrm{~Hz}$. The results show an important decrease of the fatigue limit with increasing absorbed UV irradiation dose.
\end{abstract}

\section{Introduction}

\subsection{Context of the study}

Because of their low cost, wide range of mechanical properties, and adaptability, polymers are extensively used in a large range of applications, such as aerospace and automotive industries, fluid transport, medical sectors and many others. Polymers have a large variety of molecular and supramolecular structures, separated on two basic macromolecular arrangements: amorphous and semi-crystalline structures [1]. Polyethylene (PE) is a semi-crystalline polymer that is a largely produced polymer, because of its cheap cost, outstanding electrical and mechanical properties, suitable chemical resistance, toughness, flexibility and transparency when used as thin films [2]. Polymers and particularly polyethylene microstructure and mechanical properties degrade at different rates depending on the operation environment. Studies show that polymer degradation can be induced by five processes [3]: photo-degradation by UV exposure [4], oxidation by chemical additives [5], thermal degradation by heat [6], mechanical degradation by mechanical effects [7] and biodegradation by microorganisms [8]. Depending on the environmental and operating conditions, a degradation mechanism might be preponderant relatively to the others[9]. Hence, it is necessary to identify this preponderant degradation mechanism to control through design the material's durability.

\subsection{Ultraviolet effects on polymers}

Because most of the materials operate under various weathering conditions, their lifetime must be assessed [10]. Photo-oxidation in polymers initiates a competitive degradation mechanism of chain scission versus crosslinking. Although few studied investigated the effect of UV irradiation on the mechanical behaviour of thermoplastics, fewer examined the UV irradiation effect on the fatigue life of polymers. The photo-degradation consists in the absorption of a photon of light by the polymer resulting into an alteration of the chemical and macromolecular structure of the polymer[11]. During polyolefin photo-oxidation the rates of macromolecular scission and crosslinking changes with the absorbed UV dose[12]. Chain scission mechanism is driven by the free radicals resulting from the decomposition of hydroperoxide in the presence of UV and oxygen [13]. This mechanism takes place in the amorphous phase and in the amorphous-lamellar interface [14], where the diffusion of oxygen in those phases is easier[9]. On the other hand, the crystalline phase remains typically inert [15]. This unwelcome degradation can be accelerated by increasing the absorbed UV radiation by the polymer through longer exposure time[16][17]. Several experimental techniques could be employed to point out changes of the chemical and mechanical properties undergone by polymers during and after degradation. The UV-degradation is a surface mechanism which

Corresponding author: moussa.nait-abdelaziz@polytech-lille.fr 
generally affects a thin surface layer, which thickness ranges from a few micrometres up to $1 \mathrm{~mm}$ at maximum [18]. At last, growth in crystallinity [19][20], tensile strength [21], modulus of elasticity [22], hardness [23], density [24], etc, is an indicator of the prevalence of crosslinking mechanism.

\subsection{Equivalence principle}

As polyethylene pipes are widely used in water and natural gas distribution system, they could be subjected to both UV radiations and variable mechanical loading. It is therefore important to examine the effects of UV aging on the ultimate properties and more precisely on the fatigue limit.

The time-temperature equivalence principle is generally used to build a master curve of a given material mechanical property from a set of data obtained at different temperatures or strain rates [25]. In other words, the time-temperature principle consists in making a master curve from the measurements of a mechanical property (fatigue life for example) in a specified range of temperatures (or strain rates). The resulting curves are then superimposed by shifting them via a shift factor noted $a_{T}$ that is determined from equation (1). This equivalence, suited for viscoelastic materials, allows to estimate the chosen property in the range of temperatures or strain rates experimentally unreachable (very long-time process for example). One of the most known model is the Arrhenius type law, which expresses the shift factor in the following form:

$$
a_{T}=\exp \left(\frac{E_{a}}{R T}\right)
$$

where $E_{a}$, is the effective activation energy, $\mathrm{R}$ is the gas constant, and $\mathrm{T}$ is absolute temperature.

Furthermore, when radiation (e.g., UV) is added to temperature in the aging process, this latter becomes more complex. Thermal aging leads to establishing free radicals at a thermally weak region of the polymer chains. With UV radiation, the energy involved produces more varied free radical species.

When specimen are submitted to UV irradiation, several attempted to extract an equivalence principle [25] [26][27]. The most suitable in our case is based on dose equivalency which is consistent with the concept of a limited diffusion inducing an oxidative degradation in a thin layer of the specimen [27]. In this case, the shift factor is simply expressed as follows:

$$
\begin{gathered}
a_{R}=1+R^{x} \\
t^{\prime}=\frac{t}{a_{R}}
\end{gathered}
$$

Where $\mathrm{R}$ is the dose in $\left[\mathrm{J} / \mathrm{m}^{2}\right]$ and $x$ is a parameter to be determined through the experimental results. this value being chosen to get the best fitting of the experimental data. The reduced time (or service lifetime under irradiation ageing) is expressed according to equation (3).

\section{Materials and Experimental Setups}

\subsection{Material}

The material is a low-density polyethylene (LDPE)which was supplied by QAPCOC. The physical properties of the material as provided by the supplier are as follows: Melting temperature, $109^{\circ} \mathrm{C}$; Melt Flow Index 0,30 $(\mathrm{g} / 10 \mathrm{~min})$, density $\left(0.92 \mathrm{~g} / \mathrm{cm}^{3}\right)$.

The geometry of the specimen is shown in figure 1.The fatigue samples were extracted from plates of $2 \mathrm{~mm}$ thickness. This geometry of the fatigue sample was chosen to localize the deformation in the centre of the specimen allowing fatigue crack location in the smallest cross section. Note that the radius is sufficiently large to ensure a triaxiality ratio close to that of uniaxial tension.

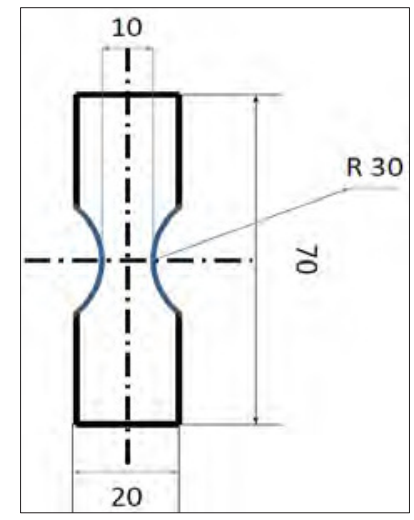

Fig. 1. Specimen geometry (dimensions in $\mathrm{mm}$ )

\subsection{Mechanical analysis}

The fatigue tests were performed on an electromechanical device (INSTRON Electro-pulse E3000) as shown in figure. 2.

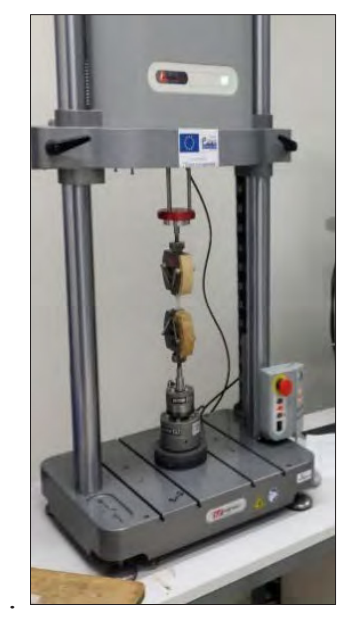

Fig. 2. Experimental setup (Instron E 3000)

The fatigue tests were achieved under force control with a $\mathrm{R}$ ratio equal to 0 . The load control condition was more 
desirable than displacement control condition as creep is induced during fatigue test (the minimum strain increases) which results into compression loading and hence buckling of the sample as shown in figure 3 .

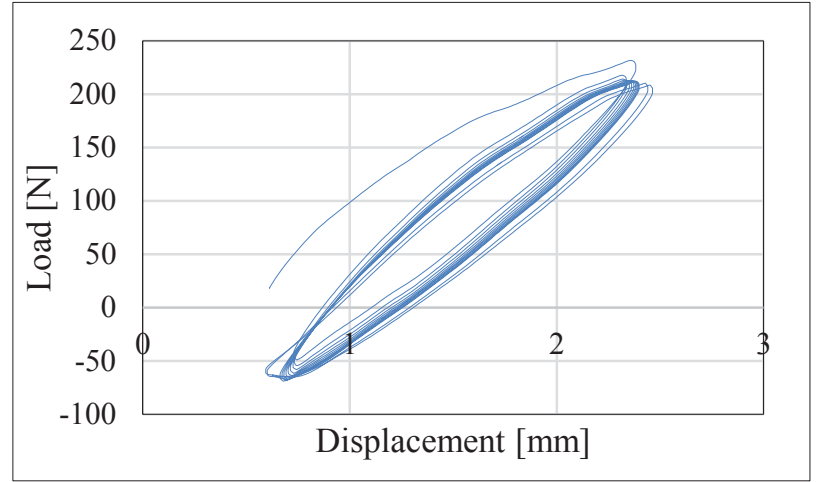

Fig. 3. Displacement control fatigue test

The tensile tests were achieved using a video extensometer (Video-Traction) device which allows keeping the strain rate at a constant value of $10^{-3} s^{-1}$ during the test. The method principle is based upon following 4 markers painted on the front surface of the sample, two marks are aligned along the tensile direction and the two other marks are aligned along the transverse direction in the minimum cross section.

\subsection{UV aging procedure}

The aging procedure consists in subjecting the specimen to UV radiations. During the procedure, exposure time is controlled in order to control the amount of energy absorbed by the specimen. A Dymax UV light-curing flood (2000) lamp was used to irradiate the specimens.
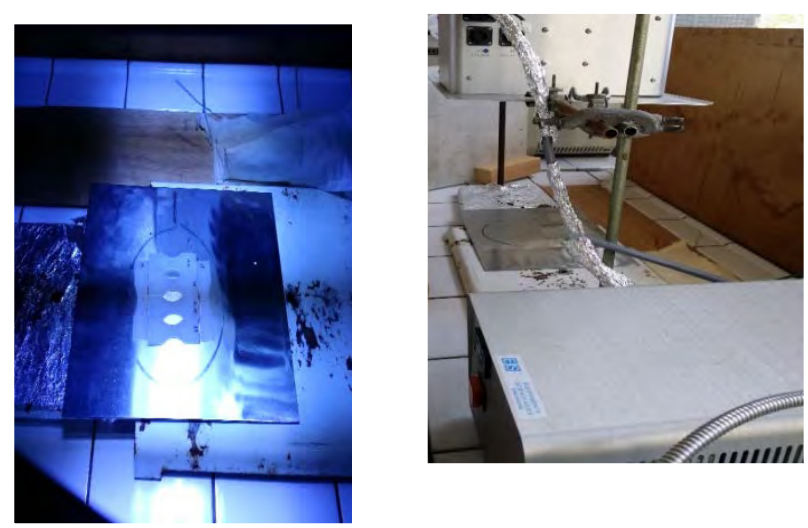

Fig. 4 .Experimental set up for UV radiations

The specimens were placed side by side under the UV lamps and exposed to irradiation (figure 4). The irradiation process is achieved in the absence of controlled temperature and humidity. Temperature measurements at the surface of the specimen raises up to $69^{\circ} \mathrm{C}$ due to the irradiation lamps. At mid-test, the specimen is turned on the other side in order to obtain the same exposure times on both sides. Table 1gives the corresponding values of the UV dose correlating with a given exposure time.
Table 1. Time exposure and corresponding UV dose

\begin{tabular}{|l|l|}
\hline $\begin{array}{l}\text { UV duration } \\
{[\text { hour }]}\end{array}$ & $\begin{array}{c}\text { Dose per side } \\
{\left[\mathrm{mJ} / \mathrm{mm}^{2}\right]}\end{array}$ \\
\hline 0 & 0 \\
\hline 5 & 580,03 \\
\hline 9 & 1038,98 \\
\hline 13 & 1497,93 \\
\hline
\end{tabular}

\section{Experimental results}

\subsection{Tensile tests}

The tensile tests were performed on as received and aged samples. The video extensometer allows to calculate the true strain and consequently the true stress in the minimum cross section. Figure 5 shows the true stress as a function of true strain for the as received material and two samples with different time exposures to UV aging. It is clearly shown that both the stiffness and the yield stress are not affected by the UV aging. The yield stress of the material is approximately $10 \mathrm{MPa}$. Nevertheless, the ultimate properties are affected by UV aging as indicated by table 2 for the strain at failure.

Table 2.Engineering strain at failure

\begin{tabular}{|c|c|}
\hline $\mathrm{UV}[$ hours $]$ & Engineering strain at failure $\%$ \\
\hline 0 & $425 \%$ \\
\hline 9 & $239 \%$ \\
\hline 13 & $95 \%$ \\
\hline
\end{tabular}

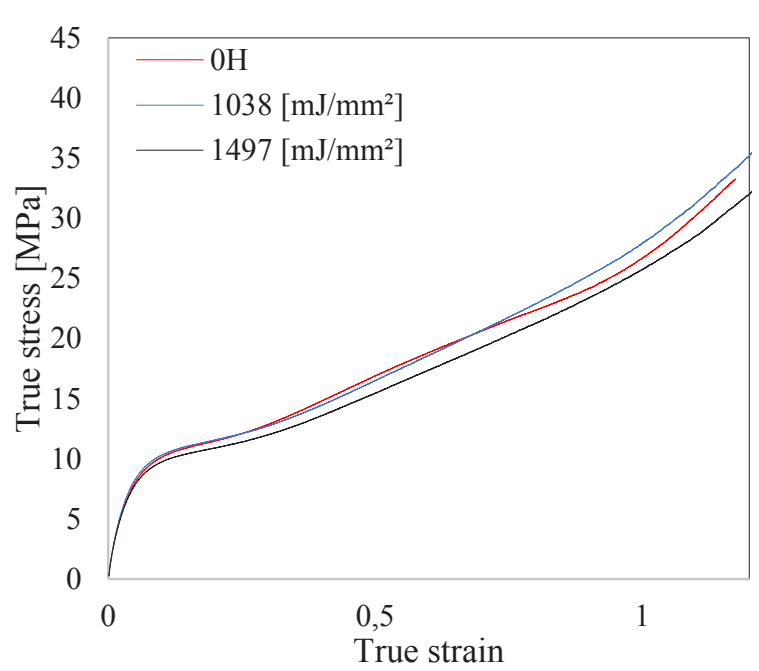

Fig. 5.Tensile tests of as received and UV-aged LDPE

\subsection{Fatigue results}

The fatigue tests under load control and constant amplitude were carried out up to the total failure of the specimens. The number of cycles to failure was therefore taken (as a first approximate) as the fatigue life. Figure 6 shows the evolution of the applied stress as a function of the fatigue life (Wohler curve). The data, plotted in a 
semi-logarithmic scale, correspond to the results obtained for 2 aging conditions: as received specimen and aged specimen (in this case, time exposure is 13 hours corresponding to an energy of $1497 \mathrm{~mJ} / \mathrm{mm}^{2}$ ).

As clearly shown, the data are quite scattered. Even scattering is generally observed when dealing with fatigue, one can note that in this case, it is relatively important. This could be the consequence of the selfheating induced by cyclic loading, the material being viscoelastic.

Nevertheless, when fitting the two set of data separately with straight lines (in the logarithmic scale), the effect of aging is clearly highlighted.

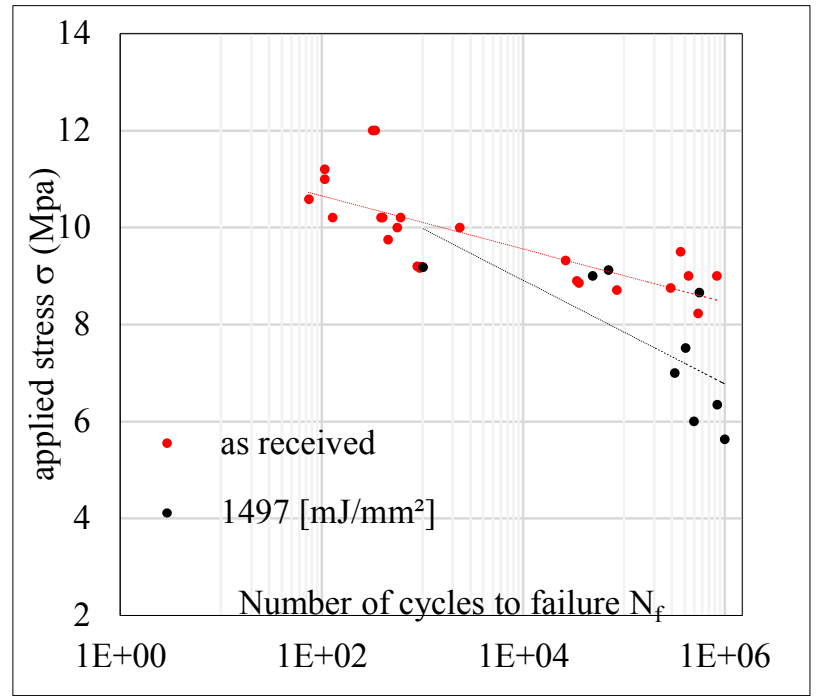

Fig. 6. Wohler curve for as received and UV-aged LDPE

Indeed, the equations of the straight lines are written in the following form:

$$
\sigma=a \cdot \log _{10}\left(N_{f}\right)+b
$$

Either the slope or the fatigue limit decreases with UV aging, as reported in the following table. This kind of behaviour was already reported in the literature but for varying $\mathrm{R}$ ratios [25]. In our case, the load ratio is constant and equal to 0. But, if we assume that UV aging induces mechanical properties change in a thin layer, then the specimen must be seen as a composite structure. Therefore, even the global $\mathrm{R}$ ratio is 0 , residual stresses acting in the layers could modify locally the value of the load ratio.

Table 3. Wohler curves slopes

\begin{tabular}{|c|c|}
\hline $\mathbf{U V}\left[\mathbf{m J} / \mathbf{m m}^{\mathbf{2}}\right]$ per side & a (MPa) \\
\hline 0 & $-0,238$ \\
\hline 1038 & $-0,355$ \\
\hline 1497 & $-0,464$ \\
\hline
\end{tabular}

Figure. 6 shows the evolution of the shift factor (equation 2) as function of the dose undergone by the specimen. In our case, the value of the parameter $\mathrm{x}$ is 6 .

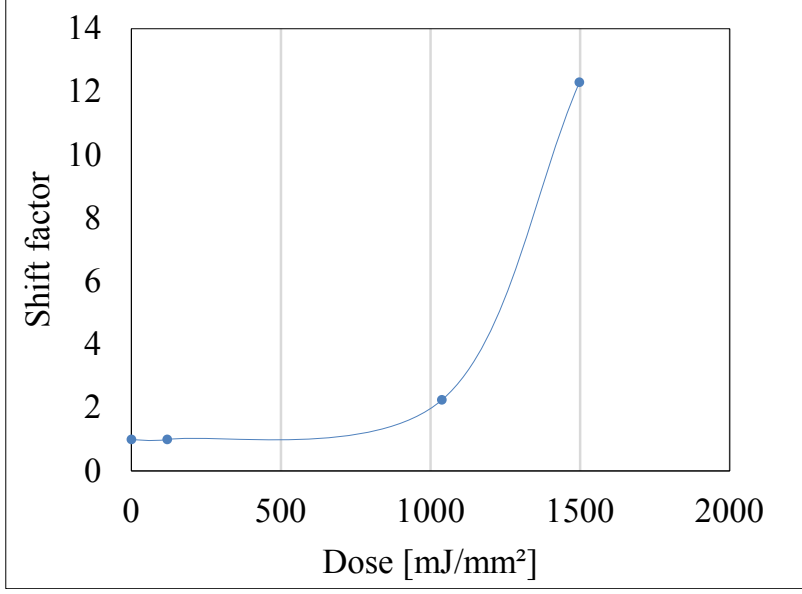

Fig. 7. shift factor function of absorbed dose

According to figure. 7, the power law evolution is an indicator of the damage severity provoked by UV radiations.

Applying the equivalence principle by multiplying the fatigue life data by the corresponding shift factor allows to obtain a master curve as shown in figure. 8 .

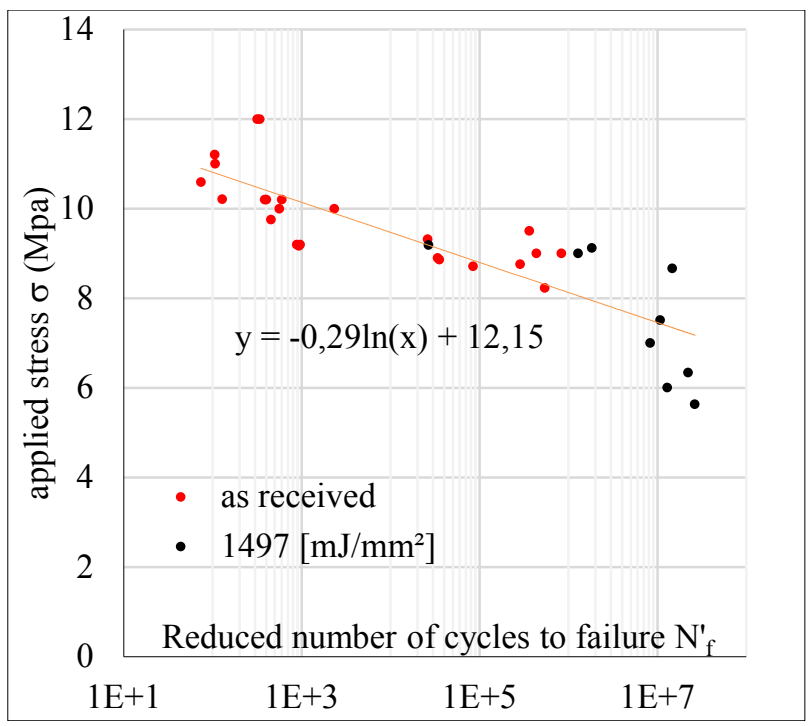

Fig. 8. Fatigue life master curve

\section{Conclusion}

In this paper, we have investigated the fatigue life behaviour of bulk low-density polyethylene submitted to UV-radiations. UV radiations generally modify the molecular structure inducing chain scission mechanism in a thin layer of the irradiated surface which thickness depends on the absorbed energy.

We have found that UV aging does not affect the mechanical response of the bulk material but the fracture properties. Indeed, stresses and strains at break decreases with increasing UV absorbed dose.

Fatigue tests were performed under load control to avoid compression loading induced by creep during fatigue cycling. We have noted an important scattering of the fatigue life data partly induced to self-heating. 
Nevertheless, the whole data can be unified using a master curve which is obtained by applying an equivalence principle based upon the radiation dose undergone by the specimen.

The decrease of the fatigue life due to aging may be explained by the existence of micro cracks initiated at the surface, which density clearly depends on the UV exposure time. Another source of the degradation of the mechanical ultimate properties could be the modification of the microstructure in the thin layer affected by UV radiations. That means the specimen behave globally as a composite structure, the mechanical properties exhibiting a gradient along the thickness.

As an outlook, this work must be completed by a deeper analysis of the fracture surface and by an estimation of the affected thickness as a function of the irradiation dose. An analysis of the microcracks in terms of size and density is also required to estimate the damage induced by UV aging.

\section{Acknowledgement}

This work was made possible by NPRP Grant \# 7-1562-2-571 from Qatar National Research Fund (A member of Qatar Foundation). The statements made herein are solely the responsibility of the authors.

\section{References}

1. Goerg H. Michler. Electron Microscopy of Polymers. (Springer Berlin Heidelberg, 2008). doi:10.1007/978-3-540-36352-1

2. Brydson, J. Plastics Materials (7th Edition) - 10 Polyethylene - Knovel. (1999).

3. Calmon-Decriaud, A., Bellon-Maurel, V. \& Silvestre, F. Standard Methods for Testing the Aerobic Biodegradation of Polymeric Materials. Review and Perspectives. in Blockcopolymers Polyelectrolytes - Biodegradation (eds. BellonMaurel, V. et al.) 207-226 (Springer Berlin Heidelberg, 1998). doi:10.1007/3-540-69191-X_3

4. Babaghayou, M. I. et al.Photodegradation characterization and heterogeneity evaluation of the exposed and unexposed faces of stabilized and unstabilized LDPE films. Mater. Des.111, 279-290 (2016).

5. Michelot, H. et al. Effect of drug precursors and chemicals relevant to clandestine laboratory investigation on plastic bags used for collection and storage. Forensic Sci. Int.273, 106-112 (2017).

6. El-Mazry, C., Ben Hassine, M., Correc, O. \& Colin, $\mathrm{X}$. Thermal oxidation kinetics of additive free polyamide 6-6. Polym. Degrad. Stab.98, 22-36 (2013).

7. White, J. R. \&Teh, J. W. Fatigue of viscoelastic polymers: 2. Fractography. Polymer 20, 764-771 (1979).
8. Kumar Sen, S. \& Raut, S. Microbial degradation of low density polyethylene (LDPE): A review. $J$. Environ. Chem. Eng.3, 462-473 (2015).

9. Kyrikou, I. \&Briassoulis, D. Biodegradation of Agricultural Plastic Films: A Critical Review. J. Polym. Environ. 15, 125-150 (2007).

10. Feldman, D. Polymer Weathering: Photo-Oxidation. J. Polym. Environ.10, 163-173 (2002).

11. Rabek, J. F. Physical aspects of the photodegradation of polymers. in Polymer Photodegradation 1-23 (Springer, Dordrecht, 1995). doi:10.1007/978-94-011-1274-1_1

12. White, J. R. \&Shyichuk, A. V. Macromolecular scission and crosslinking rate changes during polyolefin photo-oxidation. Polym. Degrad. Stab.92, 1161-1168 (2007).

13. Hsu, Y.-C. et al. A fundamental study on photooxidative degradation of linear low density polyethylene films at embrittlement. Polymer53, 2385-2393 (2012).

14. Roy, P. K., Surekha, P., Raman, R. \& Rajagopal, C. Investigating the role of metal oxidation state on the degradation behaviour of LDPE. Polym. Degrad. Stab.94, 1033-1039 (2009).

15. Koutny, M., Lemaire, J. \&Delort, A.-M. Biodegradation of polyethylene films with prooxidant additives. Chemosphere64, 1243-1252 (2006).

16. Tidjani, A. Comparison of formation of oxidation products during photo-oxidation of linear low density polyethylene under different natural and accelerated weathering conditions. Polym. Degrad. Stab.68, 465-469 (2000).

17. Şener, S. et al. World Conference on Technology, Innovation and EntrepreneurshipImpact of Solar Radiation Effects on the Physicochemical Properties of Polyethylene (PE) Plastic Film. Procedia - Soc. Behav. Sci.195, 2210-2217 (2015).

18. Cunliffe, A. V. \& Davis, A. Photo-oxidation of thick polymer samples-Part II: The influence of oxygen diffusion on the natural and artificial weathering of polyolefins. Polym. Degrad. Stab.4, 17-37 (1982).

19. Yakimets, I., Lai, D. \&Guigon, M. Effect of photooxidation cracks on behaviour of thick polypropylene samples. Polym. Degrad. Stab.86, 59-67 (2004).

20. Valadez-Gonzalez, A., Cervantes-Uc, J. M. \&Veleva, L. Mineral filler influence on the photooxidation of high density polyethylene: I. Accelerated UV chamber exposure test. Polym. Degrad. Stab.63, 253-260 (1999).

21. Kelly, C. T. \& White, J. R. Photo-degradation of polyethylene and polypropylene at slow strain-rate. Polym. Degrad. Stab.56, 367-383 (1997).

22. Carrasco, F., Pagès, P., Pascual, S. \& Colom, X. Artificial aging of high-density polyethylene by 
ultraviolet irradiation. Eur. Polym. J.37, 1457-1464 (2001).

23. Tavares, A. C., Gulmine, J. V., Lepienski, C. M. \&Akcelrud, L. The effect of accelerated aging on the surface mechanical properties of polyethylene. Polym. Degrad. Stab.81, 367-373 (2003).

24. Gulmine, J. V., Janissek, P. R., Heise, H. M. \&Akcelrud, L. Degradation profile of polyethylene after artificial accelerated weathering. Polym. Degrad. Stab.79, 385-397 (2003).

25. Guedes, R. M. Creep and Fatigue in Polymer Matrix Composites. (Elsevier, 2010).

26. Gillen, K. T. \& Clough, R. L. Time-temperaturedose rate superposition: A methodology for extrapolating accelerated radiation aging data to low dose rate conditions. Polym. Degrad. Stab.24, 137168 (1989).

27. Burnay, S. G. \&Hitchon, J. W. Prediction of service lifetimes of elastomeric seals during radiation ageing. J. Nucl. Mater.131, 197-207 (1985). 